\title{
Competência Social e Práticas Educativas Parentais em Adolescentes com Alto e Baixo Rendimento Acadêmico
}

\author{
Social Competence and Parental Practices in Adolescents with High \\ and Low Academic Achievement
}

\author{
Graziela Sapienza*, Maria Aznar-Farias \& Edwiges Ferreira de Mattos Silvares \\ Universidade Federal de São Paulo
}

\begin{abstract}
Resumo
Este trabalho investigou as relações entre competência social, práticas educativas parentais e rendimento acadêmico em adolescentes. Participaram deste estudo 66 adolescentes divididos em dois grupos: alto e baixo rendimento acadêmico. Os instrumentos utilizados foram o CBCL e o YSR (Achenbach, 1991) e o IEP (Gomide, 2006). Os resultados mostraram que adolescentes de alto rendimento acadêmico são percebidos pelos pais como socialmente competentes e são criados com mais práticas educativas parentais positivas. Esses dados indicam que o rendimento acadêmico também é influenciado pela competência social e pelo modo como os adolescentes são educados pelos pais. Esses aspectos devem ser considerados em intervenções que pretendem promover a melhoria do rendimento acadêmico e o desenvolvimento de comportamentos ajustados ao contexto escolar.

Palavras-chave: Competência social; Práticas educativas parentais; Rendimento acadêmico.

Abstract

This paper investigated the relations between social competence, parental practices and academic performance in adolescents. 66 adolescents took part in the study and they were divided in two groups: high and low academic performance. The tools used in this research were CBCL and YSR (Achenbach, 1991), and IEP (Gomide, 2006). The results showed that adolescents of high academic achievements are perceived by the parents as socially competent and are raised with more positive parental practices. The data indicated that academic performance is also influenced by the social competence and the way the adolescents are educated by the parents. Those aspects should be considered in interventions that aim at promoting the improvement of academic performance and development of a behavior adjusted to the school.

Keywords: Social competence; Parenting styles; Academic achievement.
\end{abstract}

Competência social, sucesso acadêmico e práticas parentais positivas são descritos na literatura como variáveis que promovem o ajustamento individual e interpessoal e que previnem problemas de comportamento em crianças e adolescentes. A competência social se refere à capacidade do indivíduo em manejar seu repertório de habilidades sociais (autocontrole, empatia, assertividade e habilidades para resolução de problemas interpessoais) e de informações sócio-culturais (perceber dicas do ambiente) para obter consequiências positivas para si e para sua relação com as outras pessoas (Castro, Melo, \& Silvares, 2003; Del Prette \& Del Prette, 2005; Trianes, Muñoz, \& Jiménez, 1997).

O conceito de competência social é bastante amplo e envolve a funcionalidade dos comportamentos emitidos e a avaliação dessa funcionalidade. Ser socialmente compe-

* Endereço para correspondência: Rua Euclides Bandeira, 1891, Casa 16, Centro Cívico, Curitiba, Paraná, Brasil, CEP 80530-020. Tel.: (41) 3024 5082, (41) 8837 0976. E-mail: graziela_sapienza@yahoo.com.br

Este trabalho apresenta resultados obtidos em pesquisa desenvolvida no Programa de Mestrado em Ciências da Universidade Federal de São Paulo (Unifesp), Departamento de Pediatria, Centro de Atendimento e Apoio ao Adolescente (CAAA). tente depende da efetividade e da adequação dos comportamentos emitidos à situação social em questão, o que pode ser verificado por meio das habilidades de autovalorização (autoconceito, autoconhecimento, auto-estima, auto-eficácia) e pela valorização externa, isto é, através da percepção e da reação positiva ou negativa de si mesmo e de pais, professores e pares diante do comportamento social. As habilidades de valorização podem contribuir (se positiva) ou prejudicar (se negativa) a formação do autoconceito e o incremento da auto-estima (Trianes et al., 1997).

A importância de estudar a competência social no contexto acadêmico fica evidente se consideramos que o processo de ensino e aprendizagem inclui situações de interação professor-aluno e aluno-aluno. Para realizar um exercício o estudante poderá usar o autocontrole para ouvir ou ler a instrução, a autopercepção para saber se compreendeu ou se precisa de mais informações para continuar, a assertividade para fazer perguntas ao professor ou a um colega caso seja necessário e a auto-eficácia para avaliar seu próprio desempenho naquele exercício. Se o estudante tiver um bom nível de auto-estima, um feedback negativo do professor (valorização externa) quanto ao seu desempenho 
no exercício servirá para que ele avalie seus erros e se empenhe mais em uma próxima oportunidade. Para obter êxito, além de compreender o conteúdo, o estudante precisa interagir com pares e professores e essas interações demandam um nível ajustado de competência social que se traduz em funcionamento adaptativo (Trianes et al., 1997). Ser socialmente competente contribui para o sucesso escolar já que proporciona relações positivas entre o indivíduo e os outros agentes sociais envolvidos e favorece o desenvolvimento de comportamentos necessários para aprender, como as habilidades sociais acadêmicas, o que inclui participar das tarefas em sala de aula, trocar informações, pedir correção ou orientação, aguardar a vez para falar, seguir regras e orientar-se para a tarefa (Del Prette \& Del Prette, 2005).

Mesmo diante dessas evidências, são poucos os estudos que relacionam competência social e desempenho acadêmico. Os dois temas são freqüentemente discutidos na literatura, mas separadamente. Dentre os estudos que associam competência social e rendimento acadêmico encontramos, por exemplo, que a empatia está associada a bons escores em leitura (Cotton, 2001; Papalia \& Olds, 2000) e que adolescentes com senso de auto-eficácia rebaixado possuem baixo rendimento acadêmico (Maassen \& Landsheer, 2000).

A aprendizagem dos comportamentos socialmente competentes começa nas primeiras interações familiares, com a criança sofrendo influência das práticas educativas parentais (Pacheco, Teixeira, \& Gomes, 1999). Segundo Gomide (2001), as práticas educativas parentais são as estratégias usadas pelos pais para desenvolver nos filhos a disciplina, os comportamentos sociais e estimular a independência, a autonomia e a responsabilidade, ao mesmo tempo em que modelam os comportamentos considerados por eles adequados para seus filhos. Gomide $(2001,2006)$ desenvolveu um modelo para o estudo da interação entre pais e filhos com sete práticas educativas divididas em dois grupos: as práticas educativas positivas que contribuem para o desenvolvimento do comportamento prósocial e compreendem a monitoria positiva e o comportamento moral; e as práticas educativas negativas que quando usadas freqüentemente desenvolvem o comportamento anti-social. Esse último grupo inclui a monitoria negativa, a negligência, a disciplina relaxada, o abuso físico e a punição inconsistente.

Pesquisas mostram que práticas educativas parentais positivas estão relacionadas ao desenvolvimento de comportamentos socialmente aceitos (Lubi, 2003; Pacheco et al., 1999; Patrick, 1997; Welsh, Parke, Widaman, \& O`Neil, 2001; Wentzel, 1991; Zsolnai, 2002) e a um bom rendimento escolar (Ferreira \& Marturano, 2002; Roychaudhury \& Basu, 1998). A empatia, um dos comportamentos relacionados à competência social, pode ser desenvolvida em crianças através do uso de práticas educativas parentais positivas (Motta, Falcone, Clark, \& Manhães, 2006). Práticas educativas maternas consideradas facilitadoras estão relacionadas à competência social em crianças e, ao contrário, práticas educativas não-facilitadoras estão ligadas aos problemas de comportamento externalizantes (Alvarenga \& Piccinini, 2007). Adolescentes cujos pais utilizam práticas educativas positivas, isto é, se envolvem nas vidas dos filhos, conversam e são considerados democráticos, têm melhor desempenho na escola. Adolescentes pouco valorizados por seu desempenho escolar e cujos pais utilizam práticas educativas negativas (são inconsistentes, autoritários ou negligentes e mantém interações negativas com os filhos) obtêm um desempenho escolar mais baixo, além de apresentarem condutas de isolamento e agressividade. Sendo assim, é importante considerar a influência das práticas educativas parentais na relação entre competência social e rendimento acadêmico.

A literatura mostra que a relação entre competência social, rendimento acadêmico e práticas educativas parentais é tema recente. Estudos internacionais que fazem essa relação indicam que práticas educativas positivas utilizadas por pais com estilo democrático estão relacionadas ao desenvolvimento nos filhos de comportamentos socialmente competentes e a um melhor desempenho escolar. Também mostram que práticas educativas negativas, freqüentes em pais com estilo autoritário, se relacionam aos déficits na competência social e baixo rendimento acadêmico (Chen, Dong, \& Zhou, 1997; Dawoud, 1999; Roychaudhury \& Basu, 1998).

O objetivo deste estudo foi verificar as diferenças entre competência social e práticas educativas parentais para adolescentes classificados com alto e baixo rendimento acadêmico. Para isso, duas hipóteses foram levantadas: (a) Adolescentes de alto rendimento acadêmico devem demonstrar maior competência social que os de baixo rendimento e (b) Adolescentes de alto rendimento acadêmico devem receber práticas educativas parentais mais positivas que os adolescentes de baixo rendimento.

\section{Método}

\section{Participantes}

Fizeram parte desta amostra 66 adolescentes de ambos os sexos que frequientavam uma das 11 salas de $6^{\mathrm{a}}, 7^{\mathrm{a}}$ ou $8^{\mathrm{a}}$ séries do ensino fundamental de uma escola estadual situada em Curitiba, Paraná. Esses adolescentes tinham entre 11 e 15 anos de idade. Também participaram da pesquisa os cuidadores $(n=66)$ desses adolescentes.

Os participantes foram separados em dois grupos: alto e baixo rendimento acadêmico. Para essa classificação foram utilizadas apenas as notas de português e matemática do primeiro bimestre de 2005. Essas notas foram somadas e divididas por dois, obtendo-se a média aritmética bimestral denominada "média escolar". A partir das médias escolares foram identificados os três alunos com melhor desempenho e os três alunos com pior desempenho em cada uma das onze turmas avaliadas. Os adolescentes que alcançaram as três médias mais altas $(n=33)$ foram incluídos no grupo chamado de "alto rendimento acadêmico" e os que obtiveram as três médias mais baixas $(n=33)$ no grupo chamado de "baixo rendimento acadêmico". 


\section{Instrumentos}

Child Behavior Checklist - CBCL (Achenbach, 1991). Investiga a percepção dos pais acerca da competência social de crianças e adolescentes entre 04 e 18 anos através de questões relacionadas às atividades que o adolescente desempenha (esportes, passatempos, participação em clubes e equipes, tarefas habituais), ao desempenho escolar e ao relacionamento social com amigos íntimos, irmãos, pais e pares. As respostas dos pais são inseridas em um software específico desenvolvido pelo autor que converte os resultados em $T$ escores de acordo com análises apropriadas para o sexo e a idade. Os $T$ escores são agrupados em escalas (Atividades, Social e Total de Competência Social) e os resultados dessas escalas permitem classificar os adolescentes como não-clínicos (escores acima de 41) e clínicos (escores abaixo de 40). Para este trabalho, solicitamos às mães dos alunos selecionados que respondessem ao CBCL.

Youth Self-Report - YSR (Achenbach, 1991). Versão auto-aplicável do CBCL designada para jovens entre 11 e 18 anos. A estrutura das questões e a análise dos resultados do YSR ocorrem da mesma forma como no CBCL.

Inventário de Estilos Parentais - IEP (Gomide, 2006). Investiga práticas educativas parentais relacionadas ao desenvolvimento de comportamentos pró-sociais ou antisociais em crianças e adolescentes de 9 a 18 anos. Essas práticas são divididas em dois grupos: (a) práticas educativas positivas, que envolve a monitoria positiva e o comportamento moral; e (b) práticas educativas negativas, grupo formado pela negligência, abuso físico, disciplina relaxada, punição inconsistente e monitoria negativa. Existem duas versões validadas: uma para o adolescente e outra para os pais. Neste trabalho foi utilizada apenas a versão que deve ser respondida por um dos pais. Utilizamos os dados brutos de todas as práticas educativas parentais e do IEP, obtidos através da soma das variáveis positivas (práticas positivas) menos a soma das variáveis negativas (práticas negativas). O IEP varia entre +24 e -60 e um índice positivo indica prevalência de práticas educativas positivas com pouca ou nenhuma freqüência de práticas negativas. Um escore IEP negativo é indicativo de prevalência de práticas educativas negativas com pouca ou nenhuma prática positiva. Mais informações sobre o instrumento e sua análise podem ser obtidas em Gomide (2006).

\section{Procedimentos}

Antes da coleta de dados entramos em contato com a Coordenadora Pedagógica e com a Diretora da escola para que assinassem o Termo de Consentimento Livre e Esclarecido. Após a aprovação do projeto pelo Comitê de Ética em Pesquisa da Universidade Federal de São Paulo solicitamos aos alunos de 11 salas, sendo três de sexta série, três de sétima série e cinco de oitava série que respondessem ao YSR na própria sala de aula. Aguardamos o lançamento das médias das disciplinas cursadas no primeiro bimestre de 2005 para identificarmos os alunos que participariam da amostra (conforme descrito em "participantes"). Enviamos os CBCLs e os IEPs aos pais dos alunos selecionados com instruções de como estes deveriam ser preenchidos. Os dados dos YSRs, CBCLs e IEPs foram tabulados e analisados conforme descrito a seguir.

\section{Resultados}

A análise entre os dados coletados foi realizada verificando a existência de diferenças entre as médias dos grupos de adolescentes com alto e baixo rendimento acadêmico para cada variável de competência social e de prática educativa parental. Essa diferença foi analisada pelo Teste $t$ de Student para amostras independentes.

As variáveis relacionadas à competência social e às práticas educativas parentais foram analisadas separadamente, conforme é apresentado a seguir.

A Tabela 1 mostra as diferenças entre as médias das variáveis da visão dos pais (CBCL) e da visão dos alunos (YSR) que medem a competência social para os adolescentes com baixo e alto rendimento acadêmico.

Tabela 1

Diferenças entre as Médias de Competência Social dos Adolescentes de Acordo com o Rendimento Acadêmico

\begin{tabular}{|c|c|c|c|c|c|}
\hline \multirow{2}{*}{ Variáveis } & \multicolumn{2}{|c|}{ Baixo rendimento acadêmico } & \multicolumn{2}{|c|}{ Alto rendimento acadêmico } & \multirow{2}{*}{ Teste $t$} \\
\hline & Médias & Desvio Padrão & Médias & Desvio Padrão & \\
\hline Atividades (YSR) & 44,27 & 7,39 & 45,85 & 7,16 & 0,191 \\
\hline Social (YSR) & 41,76 & 10,00 & 43,45 & 8,34 & 0,227 \\
\hline Total Competência Social (YSR) & 42,48 & 10,20 & 45,61 & 6,96 & 0,075 \\
\hline Atividades (CBCL) & 30,61 & 8,19 & 52,85 & 3,98 & $0,000 * *$ \\
\hline Social (CBCL) & 35,85 & 8,05 & 51,06 & 5,31 & $0,000 * *$ \\
\hline Total Competência Social (CBCL) & 28,15 & 8,18 & 57,27 & 8,45 & $0,000 * *$ \\
\hline
\end{tabular}

Nota. **Valores significativos para $p<0,05$

Os escores do CBCL e do YSR indicaram diferenças para os dois grupos. Os adolescentes com alto rendimento acadêmico obtiveram médias maiores em todas as variáveis que avaliam competência social, mas essas diferenças foram significativas apenas para as variáveis obtidas pelo CBCL. Os escores do CBCL mostraram que o grupo de melhor 
Sapienza, G., Aznar-Farias, M. \& Silvares, E. F. M. (2009). Competência Social e Práticas Educativas Parentais em Adolescentes com Alto e Baixo Rendimento Acadêmico.

rendimento acadêmico obteve diferenças significativas para atividades sociais $(p<0,001)$, relacionamento social $(p<0,001)$ e total de competência social $(p<0,001)$ quando comparado ao grupo de baixo rendimento acadêmico.
A Tabela 2 mostra as diferenças entre as médias das variáveis do IEP que medem as práticas educativas utilizadas pelos pais dos adolescentes de baixo e de alto rendimento acadêmico.

Tabela 2

Diferenças entre as Médias das Práticas Educativas Usadas pelos Pais dos Adolescentes de Acordo com o Rendimento Acadêmico

\begin{tabular}{lccccc}
\hline \multirow{2}{*}{ Variáveis } & \multicolumn{2}{c}{ Baixo rendimento acadêmico } & \multicolumn{2}{c}{ Alto rendimento acadêmico } & \multirow{2}{*}{ Teste $t$} \\
\cline { 2 - 5 } & Médias & Desvio Padrão & Médias & Desvio Padrão \\
\hline IEP & 5,88 & 6,82 & 8,91 & 6,71 & $0,037 * *$ \\
Monitoria Positiva & 10,97 & 1,05 & 11,36 & 1,08 & 0,069 \\
Comportamento Moral & 10,91 & 1,28 & 10,91 & 2,21 & 0,500 \\
Punição Inconsistente & 2,33 & 1,69 & 2,61 & 2,01 & 0,276 \\
Negligência & 2,67 & 2,29 & 2,52 & 1,89 & 0,385 \\
Disciplina Relaxada & 3,21 & 1,54 & 1,91 & 1,83 & $0,001 * *$ \\
Monitoria Negativa & 6,58 & 2,29 & 6,15 & 1,62 & 0,194 \\
Abuso Físico & 1,27 & 1,04 & 0,73 & 1,21 & $0,026^{* *}$ \\
\hline
\end{tabular}

Nota. **Valores significativos para $p<0,05$.

As variáveis avaliadas pelo IEP indicaram pouca diferença significativa entre o uso de práticas educativas pelos pais dos adolescentes dos dois grupos (alto e baixo rendimento acadêmico). $\mathrm{O}$ escore total do IEP foi significativamente maior entre os pais do grupo de adolescentes com alto rendimento acadêmico. Já as práticas parentais negativas denominadas disciplina relaxada e abuso físico foram significativamente menores entre os pais dos adolescentes com alto rendimento acadêmico. As outras práticas educativas negativas (punição inconsistente, negligência e monitoria negativa) não apresentaram diferenças significativas entre os dois grupos de adolescentes. Nenhuma prática educativa positiva apontou diferença significativa para os grupos de alto e baixo rendimento acadêmico.

\section{Discussão}

Partindo do princípio de que o comportamento social é parte importante do processo de ensino e aprendizagem e que o aprendizado dos comportamentos sociais se dá inicialmente na interação com os pais a partir do modo como estes educam seus filhos, optou-se por pesquisar a relação entre esses três aspectos do comportamento do adolescente: competência social, rendimento escolar e práticas educativas parentais. Essa relação foi estudada através da análise das diferenças entre competência social e práticas educativas parentais em adolescentes com alto e baixo rendimento acadêmico, a partir das duas hipóteses discutidas a seguir.

\section{Hipótese 1 - A Competência Social se Correlaciona Positivamente com o Alto Rendimento Acadêmico}

Essa hipótese foi corroborada com a análise dos dados do CBCL e do YSR, cujas médias foram mais altas nos dois questionários entre os adolescentes com alto rendimento acadêmico em todas as variáveis que avaliam competência social (Atividades, Social e Total de Competência
Social). Porém, essas diferenças foram estatisticamente significativas apenas para as variáveis obtidas pelo CBCL.

Analisando aspectos diferentes da competência social, como comportamento socialmente responsável, relacionamento com pares e empatia, encontramos na literatura que adolescentes socialmente competentes apresentam melhor desempenho escolar do que adolescentes que não se desempenham bem socialmente (Chen et al., 2003; Cotton, 2001; Maassen \& Landsheer, 2000; Wentzel, 1991). Em conformidade com os estudos citados, os dados aqui obtidos indicaram que os adolescentes com melhores notas se percebem e são percebidos pelos pais como mais competentes socialmente, quando comparados a aqueles que não estão se desempenhando tão bem nas atividades escolares.

Os dados do CBCL mostraram que os bons alunos participam de mais atividades sociais, isto é, praticam mais esportes, participam de grupos e estabelecem mais relacionamentos sociais. De ponto de vista dos pais, esses adolescentes se ajustam facilmente às demandas e expectativas das situações sociais e quando estão inseridos em algum contexto social, tendem a se desempenharem bem.

Os resultados do YSR, ainda que não tenham significância estatística, também foram importantes. A maior parte dos adolescentes dos dois grupos obteve escore acima de 41 nas variáveis relacionadas à competência social. Isso mostra que os adolescentes, independente do rendimento acadêmico, acreditam que possuem um bom repertório de comportamentos sociais, que estabelecem relacionamentos sociais adequados com pais e colegas e que participam e se desempenham bem em esportes, passatempos, tarefas rotineiras e outras atividades que envolvem grupos.

Verificou-se que as opiniões dos adolescentes de baixo rendimento acadêmico e de seus pais são diferentes em relação aos comportamentos sociais dos filhos. Os adolescentes desse grupo acreditam que se desempenham bem em atividades, mas do ponto de vista dos adultos esse desem- 
penho foi avaliado como aquém do esperado pelos pais (escore abaixo de 40). Apenas os adolescentes de alto rendimento acadêmico são vistos pelos pais como socialmente competentes nos aspectos analisados. Silva, Schoen-Ferreira, Medeiros, Aznar-Farias e Pedromônico (2004) também encontraram que adolescentes tendem a uma autopercepção positiva sobre seu comportamento.

É provável que a diferença nas visões de pais e adolescentes esteja relacionada à percepção da auto-eficácia. Os adolescentes que tiram as melhores notas também são aqueles que possuem um senso de auto-eficácia mais realista. Eles acreditam que são capazes de obter bons resultados sociais e acadêmicos provavelmente porque sabem que possuem as habilidades necessárias para isso ou, ao contrário, sabem que não as possuem, mas conseguem dispor de recursos para aprender essas habilidades. O adolescente com um nível elevado de auto-eficácia crê que é capaz, é auto-motivado e se esforça para alcançar bons resultados e atender as suas expectativas e as expectativas do meio. Quando isso não acontece, persiste para obter melhores resultados em novas tentativas. A relação entre competência social, motivação para aprender e boas notas foi mostrada anteriormente por Zsolnai (2002).

Anteriormente, Wentzel (1991) mostrou que adolescentes socialmente incompetentes apresentam também um senso de auto-eficácia rebaixado, pouca motivação principalmente para comportamentos sociais competentes e um estilo explosivo para resolução de problemas. Esses adolescentes obtêm um baixo desempenho nas atividades escolares.

Desse modo, lançamos uma pergunta: será que os adolescentes de baixo rendimento escolar obtêm notas mais baixas porque não acreditam que podem ir melhor (autoeficácia rebaixada) ou, ao contrário, porque acreditam que podem tirar uma nota maior (auto-eficácia irreal) e por isso não se esforçam tanto? Novas pesquisas devem ser realizadas para responder essa pergunta.

\section{Hipótese 2 - Há uma Associação entre as Práticas Educativas Parentais e o Desempenho Acadêmico do Adolescente}

Essa hipótese foi corroborada pelos resultados da análise dos dados do IEP. Alguns índices do IEP mostraram diferenças estatísticas significativas entre o uso de práticas educativas pelos pais dos adolescentes dos dois grupos (alto e baixo rendimento acadêmico), o índice denominado IEP que reflete o conjunto das práticas educativas utilizadas na educação dos filhos foi significativamente maior entre os pais do grupo de adolescentes com alto rendimento acadêmico.

Os resultados mostraram que os pais dos adolescentes que tiveram boas notas usam mais práticas educativas positivas e menos práticas educativas negativas, principalmente menos disciplina relaxada e menos abuso físico, do que os pais dos adolescentes de baixo rendimento acadêmico. Esses pais monitoram as atividades de seus filhos, contribuem para o desenvolvimento da auto-estima e do senso de justiça de seus filhos, tendem a manter as regras e as punições e utilizam menos punição física quando seus filhos não cumprem as regras estabelecidas. Nossos dados estão de acordo com o que também verificaram Ferreira e Marturano (2002) e Roychaudhury e Basu (1998).

As variáveis do IEP, quando analisadas separadamente, mostraram resultados interessantes. Os pais dos adolescentes de alto rendimento escolar utilizam mais a monitoria positiva e mais práticas educativas que desenvolvem o comportamento moral de seus filhos quando comparados aos pais do outro grupo. Entretanto, os pais dos adolescentes com notas mais altas também tendem a usar algumas práticas educativas negativas, como a punição inconsistente, ligeiramente mais alta do que a dos pais dos adolescentes com baixo rendimento escolar.

Os pais dos adolescentes de baixo rendimento acadêmico apresentaram mais práticas educativas negativas, principalmente disciplina relaxada, e abuso físico, quando comparados aos pais do outro grupo. Esses pais demonstram pouca atenção e afeto, têm dificuldades para responder às iniciativas dos filhos para o diálogo, estabelecem muitas regras independente de seu cumprimento, relaxam as regras já estabelecidas e punem fisicamente seus filhos, conforme indicado no IEP. Essa associação entre práticas educativas negativas e baixo desempenho acadêmico está de acordo com o que Chen et al. (1997), Maccoby e Martin (1983) e Papalia e Olds (2000) mostraram anteriormente.

\section{Conclusão}

Frente à escassez de trabalhos nacionais e à importância do tema levantada por estudos internacionais, este trabalho se propôs a analisar as relações entre competência social, práticas educativas parentais e desempenho acadêmico em adolescentes, determinando se a competência social se relaciona positivamente ao alto rendimento acadêmico e se as práticas educativas parentais estão associadas à competência social e ao desempenho escolar.

Os resultados foram compatíveis com as hipóteses, já que mostraram que adolescentes com alto e baixo rendimento acadêmico são percebidos diferentemente por seus pais e seus professores em relação à participação em atividades sociais (prática de esportes, passatempos e grupos), ao relacionamento social (interação com pares, irmãos e pais) e ao desempenho de comportamentos relacionados a esses aspectos. Adolescentes com alto rendimento acadêmico são socialmente competentes e se adaptam mais facilmente às normas escolares quando comparados aos adolescentes com baixo rendimento acadêmico.

Também foi encontrado que os pais dos adolescentes de alto e baixo rendimento acadêmico utilizam diferentemente práticas positivas e negativas para educar seus filhos. Pais de adolescentes com as notas mais altas utilizam mais práticas educativas positivas do que negativas do que os pais dos adolescentes com as notas mais baixas.

Esses resultados mostram que outros aspectos além da capacidade cognitiva são importantes para o processo de ensino e aprendizagem. Percebe-se que muitos educadores sabem disso e vêm tentando cada vez mais criar situações para que os estudantes trabalhem em grupo, o que contribui para o desenvolvimento da competência social. Porém, 
ainda parece difícil para educadores considerarem, por exemplo, a qualidade do relacionamento social como parte da avaliação de uma determinada atividade provavelmente devido às complicações para conceituar e quantificar um relacionamento ajustado para a situação.

Sabe-se que a competência social e as práticas educativas parentais estão associadas ao rendimento acadêmico. Mas existe muito a esclarecer quando se associam esses três temas. Ainda é preciso saber como essas variáveis se relacionam: será que treinar pais para que usem mais práticas educativas positivas do que negativas é suficiente para melhorar o rendimento acadêmico de seus filhos? Será que apenas desenvolver a competência social de adolescentes pode melhorar o rendimento acadêmico? Quais fatores da competência social são mais importantes para o sucesso escolar? Quais práticas educativas são mais importantes para o sucesso escolar? Essas perguntas ainda merecem resposta.

De qualquer forma, trabalhos futuros devem considerar que quando se trata de pesquisas interventivas que visem ao desenvolvimento de comportamentos socialmente competentes e à melhoria do desempenho acadêmico não basta somente trabalhar com os adolescentes, é importante incluir também os agentes sociais que influenciam os comportamentos desses adolescentes, como os pais e os professores.

\section{Referências}

Achenbach, T. (1991). Manual for the assessment data manager program (ADM): CBCL, YSR, TRF, YASR, YABCL, SCICA, $C B C L / 2-3, C B C L / 11 / 2-5 \& C-T R F$. Achenbach System of Empirically Based Assessment (ASEBA). Burlington, VT: University of Vermont.

Alvarenga, P., \& Piccinini, C. A. (2007). O impacto do temperamento, da responsividade e das práticas educativas maternas nos problemas de externalização e na competência social da criança. Psicologia: Reflexão e Crítica, 20(2), 314-323.

Castro, R. E. F., Melo, M. H. S., \& Silvares, E. F. M. (2003). O julgamento de pares de crianças com dificuldades interativas após um modelo de intervenção. Psicologia: Reflexão e Crítica, 16(2), 309-318.

Chen, X., Dong, Q., \& Zhou, H. (1997). Authoritative and authoritarian parenting practices and social and school performance in Chinese children. International Journal of Behavioral Development, 21(4), 885-873.

Cotton, K. (2001). Developing empathy in children and youth. School Improvement Research Series, Close-up \#13. Retrieved May 12, 2006, from http://www.nwrel.org/scpd/sirs/7/ cu13.html

Dawoud, N. (1999). How social competency and school antisocial behavior relate to parental socialization processes and academic achievement in a sample of 6th, 7th and 8th grade students. Dirasat: Educational Sciences, 26(1), 23-49.

Del Prette, Z. A. P., \& Del Prette, A. (2005). Psicologia das habilidades sociais na infância: Teoria e prática. Petrópolis, RJ: Vozes.

Ferreira, M. C. T., \& Marturano, E. M. (2002). Ambiente familiar e os problemas de comportamento apresentados por crianças com baixo desempenho escolar. Psicologia: Reflexão $e$ Crítica, 15(1), 35-44.
Gomide, P. I. C. (2001). Efeitos das práticas educativas no desenvolvimento do comportamento anti-social. In M. L. Marinho \& V. E. Caballo (Eds.), Psicologia clínica e da saúde (pp. 33-53). Londrina, PR: Editora da Universidade Estadual de Londrina.

Gomide, P. I. C. (2006). Inventário de Estilos Parentais - IEP: Modelo teórico, manual de aplicação, apuração e interpretação. Petrópolis, RJ: Vozes.

Lubi, A. P. L. (2003). Estilo parental e comportamento socialmente habilidoso da criança com pares. In M. Z. S. Brandão, F. C. S. Conte, F. S. Brandão, I. Ingberman, C. B. Moura, V. M. Silva, et al. (Eds.), Sobre comportamento e cognição: A história e os avanços, a seleção por conseqüências em ação (pp. 536-541). Santo André, SP: Esetec.

Maassen, G. H., \& Landsheer, J. A. (2000). Peer-perceived social competence and academic achievement of low-level educated young adolescents. Social Behavior and Personality, 28(1), 29-40.

Maccoby, E., \& Martin, J. (1983). Socialization in the context of family: Parent-child interaction. In P. H. Mussen \& E. Hetherington (Eds.), Handbook of child psychology: Socialization, personality and social development (pp. 1-101). New York: Wiley.

Motta, D. C., Falcone, E. M. O., Clark, C., \& Manhães, A. C. (2006). Práticas educativas positivas favorecem o desenvolvimento da empatia em crianças. Psicologia em Estudo, 11(3), 523-532.

Pacheco, J. T. B., Teixeira, M. A. P., \& Gomes, W. B. (1999). Estilos parentais e desenvolvimento de habilidades sociais na adolescência. Psicologia: Teoria e Pesquisa, 15(2), 117-126.

Papalia, D. E., \& Olds, S. (2000). Desenvolvimento humano (7. ed.). Porto Alegre, RS: Artes Médicas.

Patrick, H. (1997). Social self-regulation: Exploring the relations between children's social relationships, academic selfregulation, and school performance. Educational-Psychologist, 32(4), 209-220.

Roychaudhury, P., \& Basu, J. (1998). Parent-child relationship, school achievement and adjustment of adolescent boys. Journal of Personality and Clinical Studies, 14(1/2), 53-58.

Silva, M. M., Schoen-Ferreira, T. H., Medeiros, E., Aznar-Farias, M., \& Pedromônico, M. R. M. (2004). O adolescente e a competência social: Focando o número de amigos. Revista Brasileira de Crescimento e Desenvolvimento Humano, 14(1), 23-31.

Trianes, M. V., Muñoz, A. M., \& Jiménez, M. (1997). Competencia social: su educación y tratamiento. Madrid, España: Pirámide.

Welsh, M., Parke, R. D., Widaman, K., \& O’Neil, R. (2001). Linkages between children's social and academic competence: A longitudinal analysis. Journal of School Psychology, 39(6), 463-481.

Wentzel, K. R. (1991). Relations between social competence and academic achievement in early adolescence. Child Development, 62, 1066-1078.

Zsolnai, A. (2002). Relationship between children's social competence, learning motivation and school achievement. Educational Psychology, 22(3), 317-330.
Recebido: $30 / 10 / 2007$ $1^{a}$ revisão: $21 / 02 / 2008$ $2^{a}$ revisão: 06/10/2008 Aceite final: 09/10/2008 